\title{
Principales Características de un Sistema de Calibración de Torquímetros
}

Stella M. P. Domingues ${ }^{(1)}$, José L. F. Martins ${ }^{(1)}$, Rafael S. de Oliveira ${ }^{(2)}$ y Rodrigo F. Guilherme $^{(2)}$

(1) Laboratorio de Ensayos Mecánicos, Departamento de Ingeniería Mecánica,

Universidade Federal Fluminense (LEM/UFF), 108, $1^{\text {er }}$ piso del bloque E, Campus da Praia

Vermelha, Niterói-RJ, Brasil. (e-mail: stella@vm.uff.br)

(2) Laboratorio de Fuerza, División de Metrologia Mecânica, Instituto Nacional de Metrologia (LAFOR/INMETRO), Av. Nossa Senhora das Graças, 50, Xerém, Duque de Caxias, RJ-Brasil.

(e-mail: rsoliveira@inmetro.gov.br)

Recibido Jun. 24, 2009; Aceptado Ago. 11, 2009; Versión Final recibida Oct. 31, 2009

\section{Resumen}

Se definen las principales características requeridas para un sistema secundario para la calibración de torquímetros. Se describe el sistema y sus principales componentes, tales como rangos nominales, instrumento patrón utilizado, el mecanismo de aplicación del torque y la unidad digital para la adquisición de datos. Además, se presentan los principales puntos de la norma de calibración de torquímetros ISO 6789, y de esta información se realiza una evaluación de sistema prototipo existente en el Laboratorio de Pruebas Mecánicas (LEM) de la Universidad Federal Fluminense en Brasil. Aunque este prototipo ha respondido positivamente al análisis, como mejora y desarrollo del laboratorio, algunas acciones y correcciones deben ser implementadas tanto en aspectos de metrología como también en la estructura mecánica del sistema.

Palabras claves: torquímetros, calibración, par-torsional, sistemas patrón, metrología

\section{Main Characteristics of a System for Torque Wrenches Calibration}

\begin{abstract}
The main characteristics required for a secondary system for calibration of torque wrenches are defined. The system and its main components, such as nominal ranges, standard instrument used, torque applying mechanism and the digital unit for data acquisition are described. Also, the main points of the calibration standard of torque wrenches ISO 6789 arte presented, and from this information evaluation of a prototype system belonging to the Mechanical Testing Laboratory (LEM) of the University Federal Fluminense is performed. Although this prototype has positively responded to the analysis, as improvement and development of the laboratory, some actions and corrections must be implemented in metrology aspects and in the mechanical structure of the system.
\end{abstract}

Keywords: torque wrenches, calibration, torque, standard systems, metrology 


\section{INTRODUCCIÓN}

Con el crecimiento de la industria y la creciente demanda especialmente en sectores de automotriz, náutica y off-shore en gas, se hizo necesario aplicar una intensificación y la mejora del control de calidad de productos y servicios ofrecidos en apoyo de estos sectores. Entre los diversos aspectos que contribuyen a la calidad e inocuidad del producto final en estos sectores se pueden destacar la exactitud de el par torsional (torque) aplicado a la Asamblea de los equipos y estructuras (Palmeira, 2007). En las yuntas en general, la fiabilidad de estos depende de la capacidad del tornillo para fijar las piezas. La correcta configuración impide que desde el movimiento relativo entre las partes a la no pérdida de líquido, en el caso de las juntas selladas en tubos.

En el estudio de la magnitud de par, un punto importante es la diversidad de instrumentos y sistemas para las mediciones y la aplicación de esta magnitud. Podemos citar algunos ejemplos típicos de estos diferentes instrumentos, tales como transductores y torquímetros (llaves de apriete), torquímetros con palanca o axial, llaves de indicación y llaves de apriete de crack, herramientas de alto par y bajo torque, estáticas y dinámicas. Las figuras $1 \mathrm{a}$ y $1 \mathrm{~b}$ ilustran la aplicación de un torquímetro y de algunos tipos comunes de estos instrumentos, como un ejemplo.

(a)

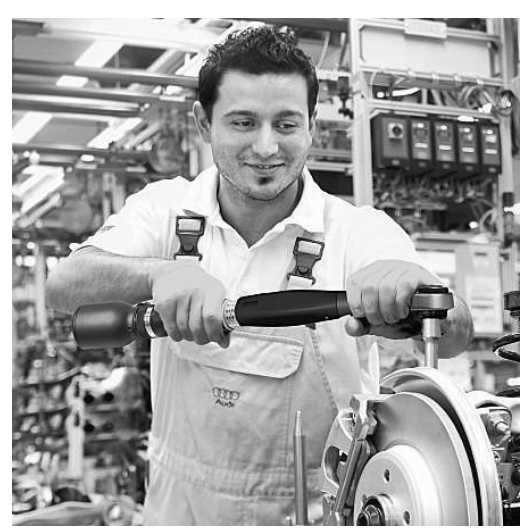

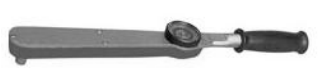

llave con relógio

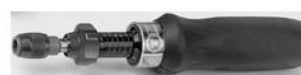

(b)

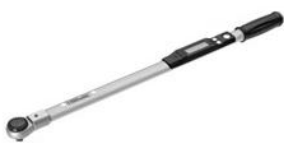

llave electrónica

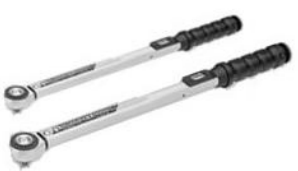

llave de 'click'

Fig. 1: (a) La aplicación de un torquímetro en un conjunto de ruedas de coche (Atlas-Copco Products, 2009); (b) Algunos tipos de torquímetros (catálogo Gedore, 2009).

En el control de calidad, estos instrumentos tienen la necesidad de ser calibrados en forma periódica de acuerdo con su uso diario y de mantenimiento después de las caídas y el desgaste posible. De ahí la necesidad de normas armonizadas de par con la fiabilidad metrológica para garantizar los resultados de estos instrumentos (Cruz, 2004; Theisen, 1997). Como parte de la metrología, son necesarios el desarrollo y adaptación de las normas y procedimientos de calibración de estos instrumentos a fin de proporcionar una mayor fiabilidad en la clasificación de estos. Con este fin, la evaluación y clasificación presentados en las normas deben ser compatibles con las características de funcionamiento, la aplicación y la posición final en la cadena de trazabilidad en metrologia de par de estos instrumentos (Cabral, 2006).

Históricamente, ha habido una evolución tanto en sistemas de calibración cuanto en los procedimientos, lo que condujo a la necesidad de establecer una metodología única para la calibración de torquímetros a calibraciones por separado de otros instrumentos medidores de par, a contener especificaciones, tolerancias, clasificación y cálculos específicos, que no se realizó hasta entonces (Incluir Imeko). Como ejemplo en Brasil, a partir de 2003 se estableció que la norma NBR 12240:00, hasta ahora la única norma de calibración en par del país, que no lograría cumplir la calibración de torquímetros, que sólo se utilizaría para los transductores de par y calibradores de torquímetros (Oliveira et al., 2003). Luego adoptó la norma ISO 6789:2003, utilizada a nivel internacional en los fabricantes de torquímetros a fin de la verificación del instrumento antes de su uso, y para calibración periódica de estos instrumentos.

La figura 2 muestra cronológicamente cómo los distintos parámetros obtenidos durante la calibración se han añadido o eliminado de los procedimientos de calibración de los torquímetros, según las 
normas de calibración se han cambiado. Así que hoy, en proyectos relacionados con aprietos mecánicos, es común que el par se determina por un valor nominal y una banda de tolerancia. Es esencial que la herramienta para la aplicación de este lío sea capaz de hacer frente a estos dos parámetros de forma satisfactoria. Así, la calibración de torquímetros tiene como principal objetivo el cálculo del error de indicación, que evalúa en qué medida el valor del torquímetro esta lejo de los valores verdaderos, y la incertidumbre de medición, que incluye principalmente los parámetros aleatorios del equipo (Inmetro, 2008; ABNT 2000).

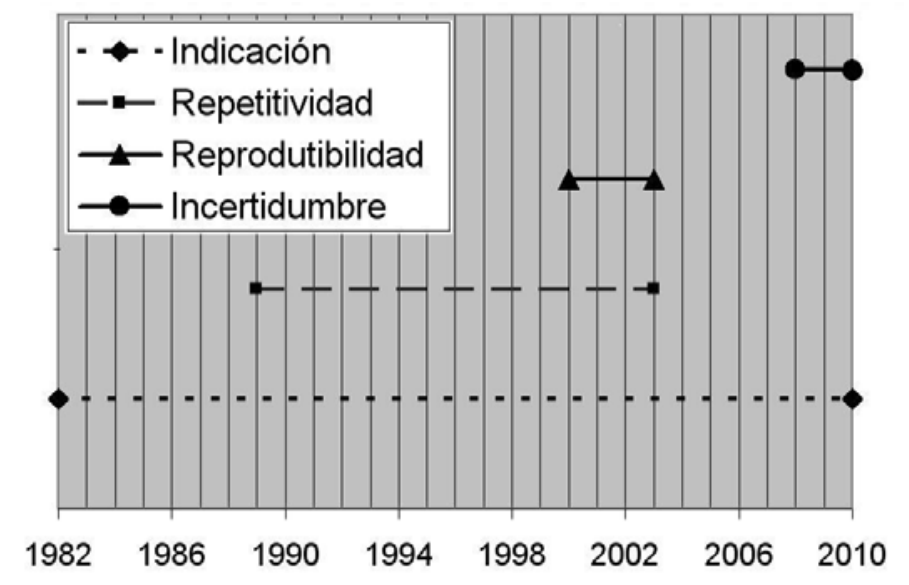

Fig. 2: Parámetros calculados para la calibración de los sistemas de calibración de torquímetros (Inmetro, 2008).

Para comprender mejor estos parámetros, la figura 3 muestra un gráfico a un rango de par específico, donde el proyecto de la junta determina que el aprieto debe tener un par nominal de 20 $\mathrm{N} \cdot \mathrm{m}$ con una tolerancia de $\pm 10 \%$. Así, la llave elegida para realizar el agarre debe tener un carácter metrológico coherente con el rango de tolerancia de diseño, es decir, el valor real de la llave de par de apriete $(X)$ debe tener una incertidumbre ampliada de medición $( \pm U)$, como no sobrepasen las tolerancias de diseño (Oliveira, 2005).

Especificacion de torsion: $20 \mathrm{~N} \cdot \mathrm{m} \pm 10 \%$

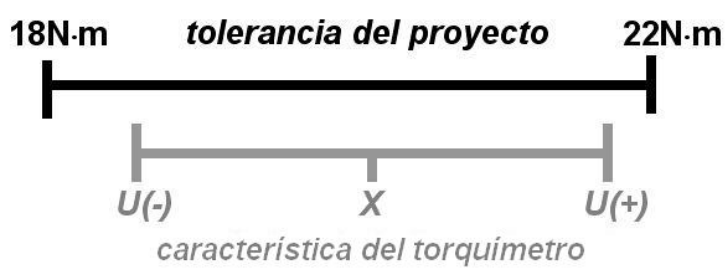

Fig. 3: Representaciones de la tolerancia de aprieto y de la incertidumbre de medición de el torquímetro.

En las secciones siguientes de este artículo se presenta, a través de la práctica y la teoría, como estos conceptos metrológicos se debe considerar y actuar a fin de determinar las principales características de un sistema de calibración de torquímetros debe tener para una correcta aplicación de la norma ISO 6789 (ISO, 2003) y de las buenas prácticas de laboratorio. Además de mostrar el análisis de un prototipo que es de construcción sencilla y económicamente viables. La ejecución de las diferentes fases de este trabajo consiste del Laboratorio de Pruebas Mecánicas (LEM) y Laboratorio de Metrología Dimensional y Computación (LMDC), ambos de la Universidad Federal Fluminense (UFF), y el Laboratorio de Fuerza y Par (Lafor) del Instituto Nacional de Metrología, Normalización y Calidad Industrial (INMETRO). El sistema de calibración propuesto se destina también a formar a los estudiantes para los cursos de licenciatura en ingeniería mecánica y ingeniería de producción, adaptándose a las exigencias actuales, con respecto a la calidad de los servicios prestados. 


\section{TRAZABILIDAD EN METROLOGIA DE TORQUE}

Según el Vocabulario Internacional de Metrología - VIM (Inmetro, 2008), la trazabilidad metrológica es "propiedad de un resultado de medición para las que este resultado puede estar relacionado con una referencia a través de una cadena ininterrumpida de calibraciones y documentada, cada uno contribuyendo a la incertidumbre de medición". La figura 4a ilustra cómo actúa la trazabilidad en términos de los patrones utilizados.

(a)

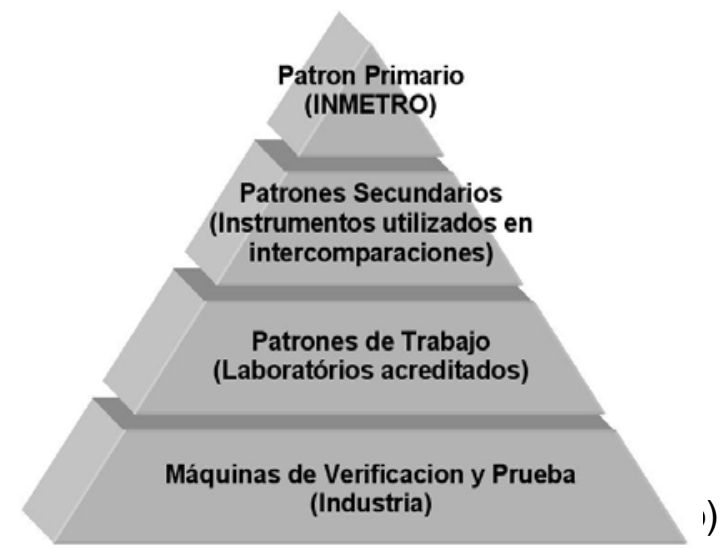

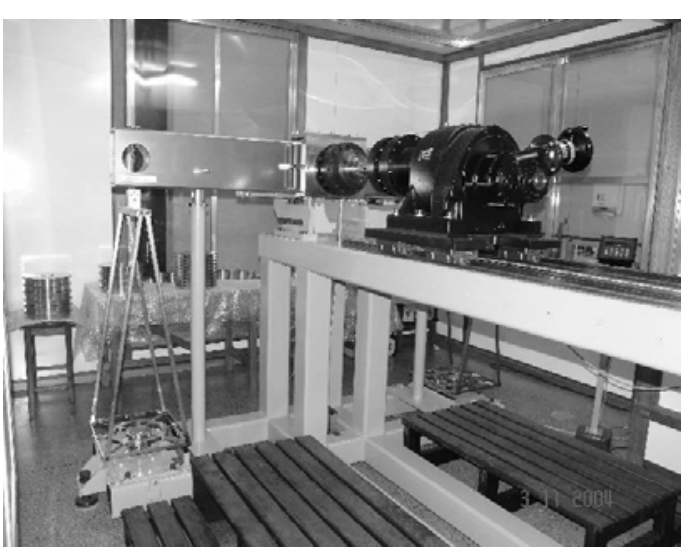

Fig. 4: (a) Pirámide de trazabilidad; (b) Patrón Primario de Par (Inmetro, 2004)

La transferencia de estas definiciones para la metrología de par, el sistema de calibración primario consta de una máquina capaz de realizar la magnitud a través del producto de la masa estándar, aceleración de la gravedad local y un brazo de referencia, en los niveles de fiabilidad y la incertidumbre es tal que reconocido internacionalmente. En Brasil, este modelo está en Inmetro (mira la figura 4b) y alcanza un valor de incertidumbre de $0,01 \%$, para la probabilidad de cobertura de $95,45 \%$, donde $\mathrm{k}=2$, de acuerdo con el guía ISO-GUM (ISO, 2003).

A través de los patrones secundarios, los valores de torque obtenidos en el nivel primario pueden ser transmitidos a los patrones de trabajo, que actuará como un estándar para la calibración de torquímetros y células de par menos precisa. La figura 5 tiene un transductor de torque (a) está calibrando en el patrón primario de Inmetro y (b) está trabajando directamente como un patrón secundario para calibrar un torquímetro.

(a)

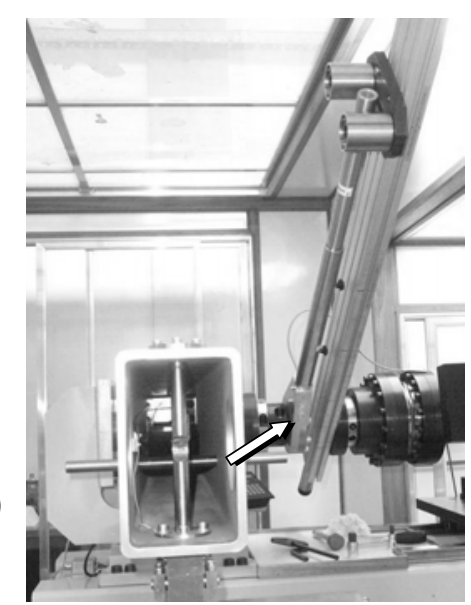

(b)

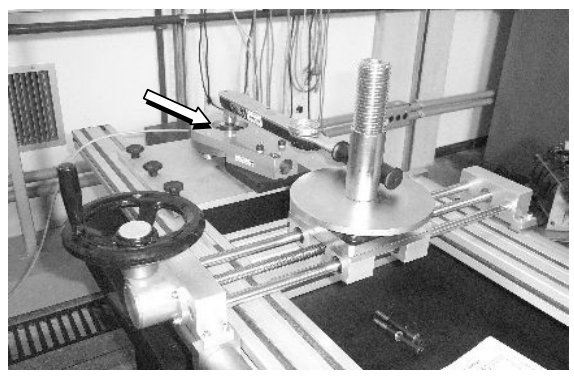

Fig. 5: (a) Transductor de par calibrado en el patrón primario, (b) transductor de par a trabajar como un patrón secundario para la calibración de un torquímetro 


\section{SISTEMAS DE CALIBRACIÓN DE TORQUIMETROS}

Mientras que la ISO 6789 no es específica acerca de qué tipo de patrón debe ser utilizado, algunas de las características esenciales y mínimas para el correcto funcionamiento de la calibración debe ser observado. El estudio de la literatura, se identificaron varios tipos de sistemas operativos con los principios y modelos diferentes. Para el análisis de un sistema, el punto de señalar en primer lugar debería ser la viabilidad de utilizar este patrón para la calibración de torquímetros y la identificación inmediata de los problemas y los parámetros que limitan la aplicación de ciertos sistemas. A menudo, los patrones utilizados en otros instrumentos de par, no es posible calibrar torquímetros. La figura 6 , la retirada de la NBR 12240 de 1989 [referencia], que versaba similarmente tanto para transductores y torquímetros, ejemplifica este punto.

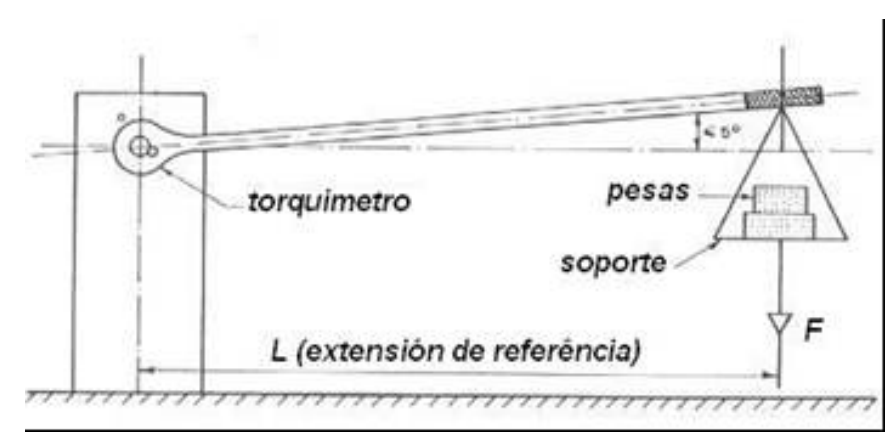

Fig. 6: sistema propuesto por la norma NBR 12240:1989 (ABNT, 1989)

En esta norma se muestra a la asamblea a calibrar el torquímetro con una aplicación de peso muerto sobre su extremidad, y tomando el brazo como la longitud de referencia, utilizando la ecuación 1 para calcular el par aplicado como patrón.

$T=m \cdot g_{1} \cdot L$

Donde: $T=$ par torsional patrón aplicado $(\mathrm{N} \cdot \mathrm{m}) ; m=$ valor total de masas $(\mathrm{kg}) ; g_{l}=$ valor de la gravedad local $\left(\mathrm{m} \cdot \mathrm{s}^{-2}\right): L=$ longitud de referencia $(\mathrm{m})$

Este sistema, aunque con el principio de la realización de una magnitud similar al método primario, es censurable para la calibración de torquímetros por varias razones, entre ellas podemos mencionar la baja resolución y baja sensibilidad en el rango de masas, la imposibilidad de utilizarlo para llaves de par de crack o axiales y el peso de la llave en sí que influye en las medidas y no se puede cancelar. Otro sistema posible es el uso de un brazo de referencia por lanzar una célula de carga. La figura 7 se muestra un esquema de cómo sería la operación de este tipo de equipos, la aplicación de un dislocación en el torquímetro y la transferencia de la fuerza (F) para el transductor de fuerza a través de la longitud del brazo (L), con el cálculo de par con la ecuación 2.

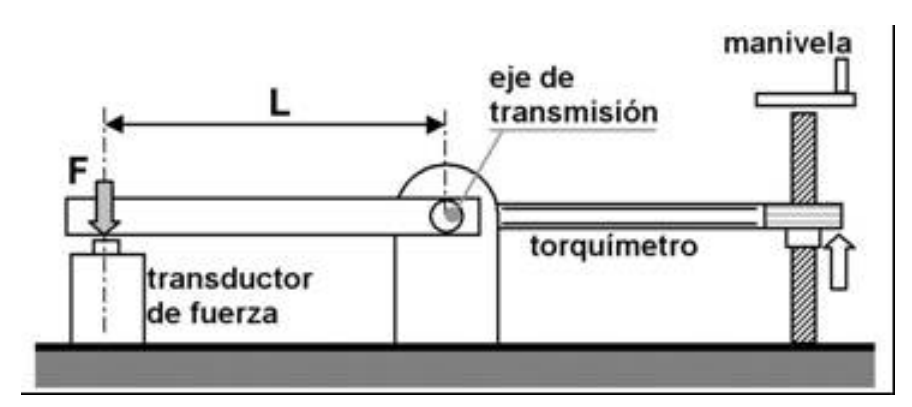

Fig. 7: sistema que utiliza un brazo de referencia y un transductor de fuerza (vista frontal) 
$T=F \cdot L$

Aunque los sistemas como éste han demostrado buenos resultados (Dantas, 2007), este proyecto tiene algunas limitaciones y la complejidad de su mantenimiento, la necesidad de mantener el eje del rodamiento para evitar cargos de la fricción; la necesidad constante de calibración además de los transductores de fuerza, el brazo de referencia para garantizar (corregir) el valor de 'L'; durante la carga el brazo no puede deformar significativamente y no causar desalineación del transductor de fuerza, escapando de los límites de tolerancia de la asamblea según la norma ISO 6789 (figura 9). Por estas razones, el sistema elegido para el estudio de caso del LEM es un prototipo compuesta por un transductor de par, de una estructura (tabla) para montar el sistema y las cargas de aplicación, y una unidad de lectura para la adquisición y entrada de valores de los instrumentos (figura 8).

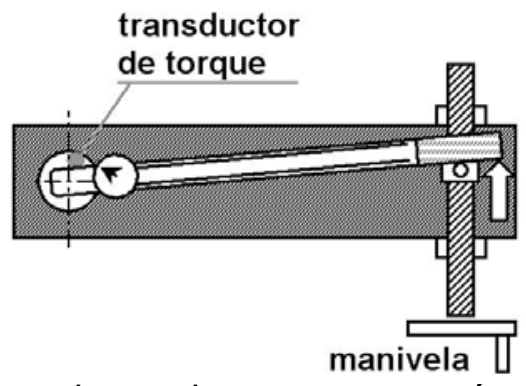

Fig. 8: prototipo con transductor de par como patrón (vista de arriba)

A continuación se presentan algunas características principales definidos, divididos en 'estructura mecánica' y 'las características metrológicas', que un sistema debe tener para llevar a cabo todo el procedimiento de calibración dado a la norma ISO 6789.

\section{Estructura mecánica}

El montaje y la estructura mecánica del sistema de calibración de torquímetros deben garantizar, especialmente, los siguientes parámetros y características:

i) Estructura inflexible para montaje y soporte de las cargas aplicadas durante la calibración pues con alta deformación (desplazamiento) de las partes juntas durante la aplicación de los torques de calibración puede provocar la aparición de parásitos esfuerzos que cambian los valores de referencia del patrón o incluso daños en el instrumentos.

ii) Sistema de fijación de diferentes transductores es común para el trabajo del sistema de calibración con varios rangos nominales diferentes, pero utilizando la misma estructura mecánica. Por lo tanto, las partes de fijar el transductor estándar debe ser intercambiables con todos los demás.

iii) Alineación de las partes en el eje de la aplicación de las cargas deben cumplir con las tolerancias se describen en la norma ISO 6789. Con este fin, la figura 9a de la norma, demuestra que el actuador de aplicación de la carga debe tener un soporte para apoyar el brazo del torquímetro y su desplazamiento no debe exceder los límites de tolerancia de la figura 9b durante la aplicación del torque. Es ideal que el torquímetro esté en posición horizontal en el banco, a fin de eliminar la influencia de su propio peso para obtener el cero en la serie de medición.

iv) La distancia entre la "cabeza" del torquímetro y el punto de aplicación del actuador debe ser variable para adaptar el tamaño de los diferentes brazos de torquímetros.

v) El sistema debe permitir la aplicación de la carga en ambas direcciones, derecha y izquierda, en una sencilla y práctica, de preferencia sin interferir con el re-montaje de los patrones. 
vi) La aplicación de la carga puede ser manual o automático, a condición de que se trata de una variación de la velocidad desde el inicio de la aplicación hasta el enfoque del punto de medición. El operador responsable de la aplicación de los torques debe ser capaz de garantizar también el mantenimiento de la carga en los puntos de medición en las secuencias de cargamento.

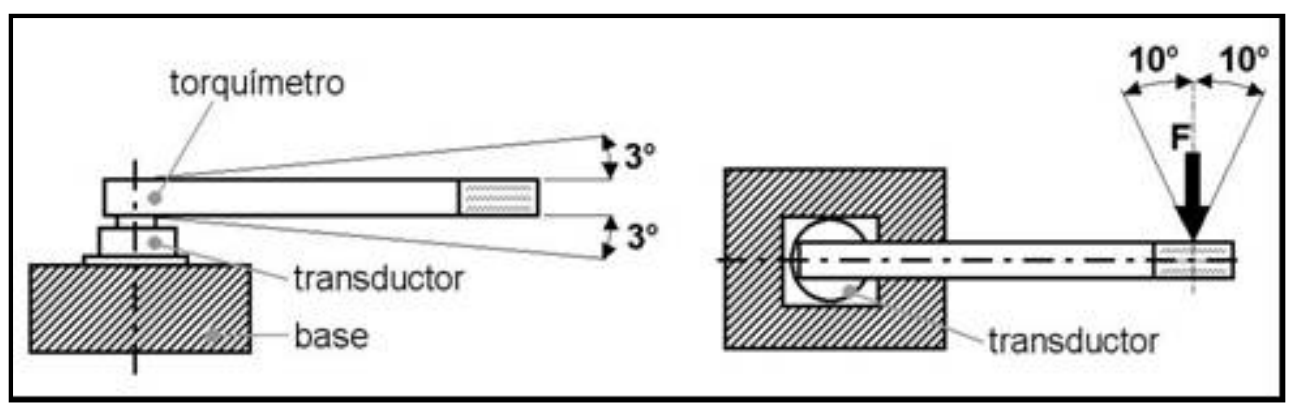

Fig. 9: Tolerancias dimensionales para el montaje de los torquímetros (ISO, 2003).

\section{Características metrológicas del transductor}

Todos los transductores y el indicador digital debe ser calibrado con arreglo a procedimientos internacionalmente reconocidos y el único requisito de la norma ISO 6789:2003 es la incertidumbre de medición de el patrón es menor o igual a $\pm 1 \%(k=2)$ para los puntos ser calibrado del torquímetro. Como ejemplo de este cálculo, la ecuación 3 siguiente muestra la pertinencia, a través de la ley de propagación de incertidumbre (ISO, 2003), los parámetros que contribuyen para el cálculo de la incertidumbre de medición de los transductores de torque, de conformidad con el guía de calibración Euramet/cg-14/v.01 "Directrices para la calibración de dispositivos estáticos de medición de par" (Euramet, 2007).

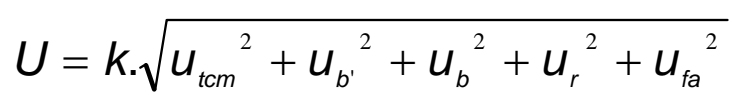

Donde: $u_{t c m}=$ incertidumbre del torque de referencia; $u_{b^{\prime}}=$ incertidumbre relativa a la repetibilidad; $u_{b}$ $=$ incertidumbre relativa a la reproducibilidad; $u_{r}=$ incertidumbre relativa a la resolución del indicador; $u_{f a}=$ incertidumbre relativa a la curva de interpolación; $k=$ factor de cobertura para la probabilidad de $95,45 \%$

Por lo tanto, para la evaluación metrológica del transductor y de su viabilidad como patrón, estos parámetros deben trabajar para cumplir los requisitos de la norma. Cabe señalar que el valor máximo de $1 \%$ especificado en la norma debería abordar específicamente el punto de calibración, o si algún otro punto del transductor que supere ese límite, pero no utilizado para la calibración, no invalida el resto del rango. Del mismo modo, si un patrón único no es suficiente para satisfacer todo el rango de calibración del torquímetro, otro patrón complementario debe ser utilizado. Dentro de este conjunto de parámetros, puede presentarse como mas critico la reproducibilidad, sobre la capacidad reproductiva de los valores en diferentes condiciones de carga, y la curva de interpolación (o curva de ajuste) del transductor, por lo general relacionadas con su linealidad.

\section{ANALISIS DEL PROTOTIPO}

El prototipo fue diseñado para una carga máxima de $300 \mathrm{~N} \cdot \mathrm{m}$, capacidad nominal del transductor y una de las bandas de mayor demanda en el mercado para el uso y calibración de los equipos. La figura 10 muestra la tabla durante la aplicación de la carga. El transductor utilizado también es de fabricacion del mismo laboratorio, a través de la instrumentación con el uso de strain-gages, especificos para lectura de torque, fijo en el cuerpo del transductor, aislados y protegidos. Se utilizó una configuración de puente completo con la salida a 6-pin plug, por lo tanto, el transductor puede ser conectado tanto a los indicadores digitales de manera más precisa y en los indicadores mas simples. 
El análisis del prototipo se realizó en tres etapas, inicialmente evaluar la conducta de la Asamblea mecánico, posteriormente las características metrológicas del transductor y una auditoria de medición con la calibración de un torquímetro como artefacto.Para el primer análisis, basado en las características detalladas en la sección anterior, un torquímetro de $20 \mathrm{~N} \cdot \mathrm{m}$ (aprox. $200 \mathrm{~N} \cdot \mathrm{m}$ ) se montará en el sistema y algunos puntos de torque se han aplicado. La tabla 1 presenta una lista de las principales características mecánicas y la evaluación del funcionamiento del prototipo para cada uno, y una columna de comentarios para observaciones y diagnósticos inmediatos para ese parámetro.

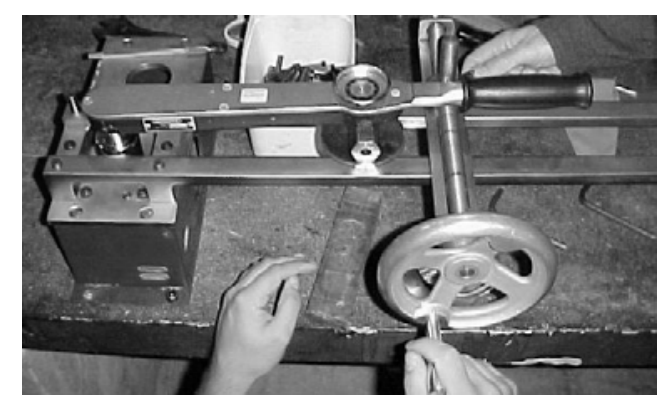

Fig. 10: foto del prototipo y del transductor.

Tabla 1: Análisis de la estructura mecánica y funcionalidad de la asamblea

\begin{tabular}{|c|c|c|c|}
\hline Característica (parámetro) & \multicolumn{2}{|c|}{ Respuesta } & Observaciones \\
\hline Estructura rígida & 区lok & $\square$ no & La estructura no mostró deformación durante la aplicación de torque \\
\hline $\begin{array}{l}\text { Mantener el valor del torque } \\
\text { aplicado }\end{array}$ & $\square$ ok & 凶no & $\begin{array}{l}\text { Después del punto de } 250 \mathrm{~N} \cdot \mathrm{m} \text {, el sistema no se mantenga la carga } \\
\text { aplicada en un tiempo mínimo para la lectura. }\end{array}$ \\
\hline $\begin{array}{l}\text { Sistema de fijación de diferentes } \\
\text { transductores }\end{array}$ & $\square$ ok & Qno & $\begin{array}{l}\text { Aunque la caja de encaje del torquímetro es extraíble, el espacio } \\
\text { para la asamblea no es compatible con cualquier tipo de transductor. }\end{array}$ \\
\hline $\begin{array}{l}\text { alineación del torquímetro dentro de } \\
\text { las tolerancias }\end{array}$ & 囚ok & $\square$ no & $\begin{array}{l}\text { Durante la aplicación de torque, la deformación del elemento elástico } \\
\text { del transductor no se mueve el torquímetro más allá de las } \\
\text { tolerancias de norma. El torquímetro se mantuvo en la posición } \\
\text { horizontal. }\end{array}$ \\
\hline $\begin{array}{l}\text { Distancia variable para la aplicación } \\
\text { de la fuerza y aplicación de par en } \\
\text { ambas direcciones }\end{array}$ & 囚ok & $\square$ no & $\begin{array}{l}\text { La extensión máxima alcanzada por el actuador hasta la cabeza del } \\
\text { torquímetro es de } 90 \mathrm{~cm} \text {, muy bueno para la gama de par deseado. } \\
\text { Actuador funciona en ambas direcciones. }\end{array}$ \\
\hline Ergonomía & 囚ok & $\square$ no & $\begin{array}{l}\text { El operador puede aplicar carga y hacer la lectura en el visor del } \\
\text { torquímetro simultáneo }\end{array}$ \\
\hline
\end{tabular}

De acuerdo con la tabla 1, la estructura mecánica tiene deficiencias en el mantenimiento de las cargas aplicadas y el cambio poco práctica del patrón a utilizar. El primer problema que debe ser resuelto con poner una caja de cambios entre la manivela y el eje de movimiento del actuador, para no permitir que el par de reacción mueva el actuador en el punto de medición de torque. Para resolver el segundo problema, se propone cambiar el sistema de apareamiento de los transductores de una caja a una tabla (placa de apoyo), donde el transductor es fijo y el espacio para su uso no se limita las dimensiones de la caja. Para ello, las guías se fijará en la placa del actuador y la caja, que se adjuntaba el transductor será eliminado.

Para el examen de las características metrológicas, sobre la segunda análisis del prototipo, el transductor ha sido calibrado en un sistema simple que consiste en masas calibrado y un brazo de palanca, también calibrado, para generar el par de referencia. Aunque el principio de funcionamiento es similar a el patrón primario de masa, brazo y valor de la gravedad local, este conjunto no tiene la misma capacidad de medición, y la incertidumbre se determina como $U=0,3 \%$. La tabla 2 muestra los principales parámetros obtenidos de esta calibración. 
Tabla 2: Parámetros da calibración

\begin{tabular}{|l|l|}
\hline Característica (parámetro) & \multicolumn{1}{c|}{ Descripción } \\
\hline Sistema de referencia & $\begin{array}{l}\text { Masas calibradas, Brazo de referencia }(1 \mathrm{~m}) \text { e Valor da gravedad local } \\
\left(9,7874867 \mathrm{~m} \cdot \mathrm{s}^{-2}\right) \\
\text { Incertidumbre expandida } U=0,3 \%\end{array}$ \\
\hline Indicador digital & $\begin{array}{l}\text { Indicador digital especial para transductores, con voltaje de 10V e rango de } \\
\text { indicación en } \mathrm{mV} / \mathrm{V}\end{array}$ \\
\hline Resolución del indicador & $0,0001 \mathrm{mV} / \mathrm{V}$ (debido a la inestabilidad, aprobó la resolución de 0,0002 $\mathrm{mV} / \mathrm{V}$ ) \\
\hline Series de medición & $\begin{array}{l}3 \text { precargas, 2 series en la posición inicial, mas 1 serie con la montaje } \\
\text { modificada. } \\
\text { Pontos atingidos pelo conjunto de masas }=10,20,39,59,108,157,254 \mathrm{~N} \cdot \mathrm{m}\end{array}$ \\
\hline
\end{tabular}

Aunque la pantalla del indicador digital de hasta 4 cifras decimales y el aumento de 1 dígito en la última casa, la norma de referencia para la calibración de transductores (Euramet, 2007) establece que la resolución adoptada para los cálculos es la mitad de la variación de cifras con una carga nula. La tabla 3 muestra los principales resultados de la calibración y el valor de la incertidumbre para cada punto, calculado de acuerdo a la pertinencia ecuación 4.

Tabla 3: contribuciones de incertidumbre de cada parámetro y el valor final de incertidumbre expandida (dados en \%).

\begin{tabular}{|c|c|c|c|c|c|}
\hline $\begin{array}{c}\text { Rango de } \\
\text { calibración } \\
N \cdot m\end{array}$ & $\begin{array}{c}\text { Repetibilidad } \\
\left(u_{b^{\prime}}\right)\end{array}$ & $\begin{array}{c}\text { Reproducibilidad } \\
\left(u_{b}\right)\end{array}$ & $\begin{array}{c}\text { Interpolación } \\
\text { lineal }\left(u_{f a}\right)\end{array}$ & $\begin{array}{c}\text { Resolución } \\
\left(u_{r}\right)\end{array}$ & $\begin{array}{c}\text { Incertidumbre } \\
\text { expandida } \\
\text { para } k=2(U)\end{array}$ \\
\hline 10 & 0,4835 & 0,1712 & $\mathbf{0 , 6 4 1 9}$ & 0,0989 & 1,68 \\
\hline 20 & 0,0000 & 0,0845 & $\mathbf{0 , 1 2 5 9}$ & 0,0488 & 0,44 \\
\hline 39 & 0,0596 & $\mathbf{0 , 0 6 3 3}$ & 0,0485 & 0,0244 & 0,36 \\
\hline 59 & 0,0199 & 0,0000 & $\mathbf{0 , 0 3 4 1}$ & 0,0162 & 0,31 \\
\hline 157 & 0,0075 & 0,0053 & $\mathbf{0 , 0 1 6 2}$ & 0,0061 & 0,30 \\
\hline 254 & 0,0046 & $\mathbf{0 , 0 0 6 5}$ & 0,0064 & 0,0037 & 0,30 \\
\hline
\end{tabular}

Los datos en negrita en la tabla indican los parámetros con una contribución mayor al valor de la incertidumbre final en cada punto del rango de calibración excluso la influencia de la incertidumbre del sistema de calibración $\left(u_{t c m}\right)$, predominante en los últimos tres puntos. Por lo tanto, la reproducibilidad y la interpolación lineal se confirman como los parámetros de mayor influencia en la cantidad de incertidumbre.

En cuanto a la última columna con los valores de incertidumbre expandida, se observa que, excepto el primer punto de $10 \mathrm{~N} \cdot \mathrm{m}$, todos los otros estaban dentro de límites tolerables de $\pm 1 \%$ de la norma ISO 6789 para un transductor se utiliza como estándar referencia a los torquímetros. A fin de mejorar estos valores de incertidumbre se propone llevar a cabo una calibración del transductor en el sistema primario del Inmetro, que tiene la incertidumbre es de 0,01\%.

Para un análisis final de la viabilidad y caracterización del sistema propuesto, se realizó una auditoria de medición, con la calibración de un torquímetro de indicación del tipo de reloj hasta $20 \mathrm{kgf} \cdot \mathrm{m}$ $(196,133 \mathrm{~N} \cdot \mathrm{m})$, en los sistemas de INMETRO y del LEM según la norma ISO 6789, es decir, el total de tres puntos con cinco cargas de cada uno. El sistema fue utilizado en Inmetro un transductor (véase la figura $5 \mathrm{~b}$ ) de rango nominal de $3000 \mathrm{~N} \cdot \mathrm{m}$ y la incertidumbre ampliada de $0,2 \%$ en los puntos de calibración del torquímetro. La siguiente tabla 4 muestra el promedio final y la incertidumbre, $X$ y $U$, y el valor del error normalizado $\left(E_{N}\right)(A B N T, 1999)$ calculado para cada punto del rango. 
Tabla 4: Datos de la auditoria de medición

\begin{tabular}{|c|c|c|c|c|c|c|}
\hline \multicolumn{2}{|c|}{ Faixa calibrada } & $X_{R}$ (Inmetro) & $X_{L}($ LEM) & $U_{R}$ (Inmetro) & $U_{L}($ LEM) & \multirow{2}{*}{$E_{N}$} \\
\hline Kgf $\cdot \mathrm{m}$ & $\mathrm{N} \cdot \mathrm{m}$ & \multicolumn{3}{|c|}{$\mathrm{N} \cdot \mathrm{m}$} & 4,02 & 0,15 \\
\hline 4 & 39,226 & 42,23 & 41,37 & 4,02 & 4,03 & 0,40 \\
\hline 12 & 117,679 & 116,64 & 114,38 & 4,03 & 4,07 & 0,72 \\
\hline 20 & 196,133 & 195,19 & 191,03 & 4,13 & \\
\hline
\end{tabular}

El análisis estadístico de error normalizado (la ecuación 4) afirma que esto debe ser inferior a una unidad de modo que es positivo, lo que realmente sucedió en todos los puntos del rango.

$E_{N}=\frac{X_{L}-X_{R}}{\sqrt{U_{L}^{2}+U_{R}^{2}}}$

La ecuación 5 para calcular la incertidumbre de torquímetros fue desarrollado a partir del guía ISOGUM y muestra una gran influencia de la resolución del torquímetro, en este caso determinado como la mitad del valor de una división, o $0,5 \mathrm{kgf} \cdot \mathrm{m}$, que tiene una homogeneización análisis sobre la comparación de ambos los sistemas.

$U=k \cdot \sqrt{u_{t c m}^{2}+\left(\frac{\text { desvpad }}{\sqrt{5}}\right)^{2}+\left(\frac{\text { resol }}{\sqrt{6}}\right)^{2}}$

\section{CONCLUSIONES}

Aunque los resultados finales del análisis de la auditoria de la medición ha sido positiva para la viabilidad del sistema, el personal de LEM, a lo largo de las directrices del Inmetro, concluye son necesarias acciones destinadas a mejorar el sistema. Al final del análisis de la estructura mecánica y de las características metrológicas del transductor en el prototipo, algunas de estas acciones ya se han explicado. A continuación se presentan otros servicios complementarios a las siguientes:

a) Calibración del transductor en un sistema estándar de referencia más precisa (del Inmetro);

b) Evaluación de la estabilidad del transductor en largo plazo (verificación periódica);

c) Uso de torquímetros de diferentes rangos, tipos y exactitud en las auditorias de medición;

d) Sistema de lectura más práctico, preciso y estable, como las tarjetas de adquisición a través de $\mathrm{PC}$;

e) Un mejor modelado en el cálculo de la incertidumbre de medición, mientras que los parámetros más influyentes;

f) Adecuación de las condiciones ambientales del LEM.

Con la aplicación de calibración en par, el LEM es capaz de realizar las principales pruebas (mecánico) en el área de fuerza (tracción, compresión, flexión plana y fatiga), la dureza, el impacto y la torsión. Esta expansión de las pruebas mecánicas permite el LEM contribuye a la formación de estudiantes de postgrado, dejándolos mejor preparados para la realidad del mercado. Además de colaborar en la formación de los estudiantes, la expansión de las pruebas de ayuda para prestar servicios a otros laboratorios de la Escuela de Ingeniería en el ámbito de la educación y la investigación y la prestación de servicios a las empresas de fuera de la UFF, permitiendo la captación de recursos propios para el mantenimiento y la modernización de laboratorio.

\section{REFERENCIAS}

ABNT ISO GUIA 43:1999 partes 1 e 2, "Ensaios de Proficiência por Comparações Interlaboratoriais", Associação Brasileira de Normas Técnicas, Brasil (1999).

ABNT NBR 12240, "Aferição de Torquímetros", Associação Brasileira de Normas Técnicas, Rio de Janeiro,1989. 
ABNT NBR 12240, "Materiais metálicos - Calibração e classificação de instrumentos de medição de torque", Associação Brasileira de Normas Técnicas, Rio de Janeiro, 2000.

ABNT NBR ISO/IEC 17025, "Requisitos Gerais para Competência de Laboratórios de Ensaio e Calibração", Associação Brasileira de Normas Técnicas (2005).

Atlas Copco Products, [fecha de consulta 15 Agosto 2009], www.directindustry.com.

Catálogo Productos Gedore, [fecha de consulta 15 Agosto 2009], www.gedore.com.br.

Cruz, V.A., "Sistema de Gestión de la Calidad en el Apoyo a la Implementación de Estrategias de Producción Ajustada", Información Tecnológica-Vol. 15 N6-2004, págs.: 63-70.

Dantas, A.B., "Projeto e Construção de Padrão de Torque para Calibração de Torquímetros em Três Escalas", Maestria, Dpto. Ing. Mecánica, Universidade Federal do Rio Grande do Norte, Brasil (2007).

EURAMET/cg-14/v.01, "Guides on the Calibration of Static Torque Measuring Devices", European Accreditation (2007).

Ferrero, C., "Dissemination of the Torque Unit in Italy: Intercomparison Results on Torque Wrenches", XVII IMEKO World Congress, Dubrovnik, Croatia (2003).

Freitas, L.C.C., "Implantação da padronização primária de torque no Brasil”, Maestria, Dpto. Ing. de Producción, Universidade Federal Fluminense, Brasil (2006).

INMETRO, Inst. Nac. de Metrología, Normalização e Qualidade Ind., "VIM - Vocabulário Internacional de Metrologia - Conceitos Fundamentais e Gerais e termos asociados", Rio de Janeiro, Brazil (2008).

ISO 6789:2003, "Assembly tools for screws and nuts - Hand torque tools - Requirements and test methods", Geneva, Suiça (2003).

ISO-GUM, "Guia para a Expressão da Incerteza de Medição - Terceira Edição Brasileira do "Guide to the Expression of Uncertainty in Measurement", 3a edição, ISO, IEC, BIPM, OIML, EA, NAMAS/UKAS, INMETRO (2003).

Oliveira, R.S., "Apostila do Curso Metrología de Torque e Normas de Calibração", INMETRO, Rio de Janeiro, Brazil (2005).

Oliveira, R.S., L.C.C. Freitas y J.A.P. Cruz, "Interpretação e Aplicação das Normas NBR 12240 e ISO 6789 na Calibração de Instrumentos de Medição de Torque", Congresso Metrologia 2003, Recife, Brazil (2003).

Palmeira, A.M., S.M.P. Domingues, R.S., Oliveira y J.L.F., Martins, "Projeto de Sistemas Secundário de Calibração de Torquímetros do LEM/UFF-Brasil", 8 Congresso Iberoamericano de Engenharia Mecanica, Cusco, Peru (2007).

Theisen, A.M. de F., "Fundamentos de Metrología Industrial - Aplicação no Processo de Certificação ISO 9000", SEBAE-LABELO-FIERGS, Brasil (1997). 